Highly Energetic Physical Processes and

Mechanisms for Emission from Astrophysical Plasmas

IAU Symposium, Vol. 195, 2000

P. C. H. Martens, S. Tsuruta, and M. A. Weber, eds.

\title{
A Three-dimensional Outer-magnetospheric Gap Model for Gamma-ray Pulsars: I. The Crab Pulsar
}

\section{K. S. Cheng}

Department of Physics, the University of Hong Kong, P.R. China

\author{
M. Ruderman
}

Department of Physics, Columbia University, U.S.A.

L. Zhang ${ }^{1}$

Department of Physics, the University of Hong Kong, P.R. China

\begin{abstract}
We use a three-dimensional pulsar magnetosphere model to study the geometry of outer-magnetospheric gaps. The vertical size of the "outer gap" is first determined by a self-consistent model in which the outer gap size is limited by pair production from collisions between (1) thermal photons produced from polar cap heating by backflow "outer gap" current, and (2) the curvature photons emitted by gap-accelerated charged particles. The transverse size of the outer gap is also determined by local pair production limits. In principle, there are two topologically disconnected outer gaps in the magnetosphere of a pulsar. Both incoming and outgoing particle flows are allowed. However, the emission morphologies produced by incoming particle flow is severely restricted by local pair production in the gap and the absorption of magnetic pair production near the star. Double-peaked light curves with strong bridges are most common. From the three-dimensional structure of the outer gap and its local properties, we calculate the emission morphologies and phase-resolved spectra of gamma-ray pulsars. Applications to the Crab pulsar illustrate the model.
\end{abstract}

\section{Introduction}

It has been argued that powerful acceleration regions, called "outer gaps", can form in the vicinity of "null charge surface" $(\boldsymbol{\Omega} \cdot \mathbf{B}=0)$ (Holloway 1973; Cheng, Ruderman, \& Sutherland 1976) because the charged carriers on each side of the null charge surface have opposite charges. Current passing through this surface remove charges in the vicinity of the null surface, and a vacuum will form there. Cheng, Ho, \& Ruderman (CHR I; CHR II) used an outer gap to calculate the phase-averaged spectrum of the Crab pulsar. Their model assumed that the ra-

\footnotetext{
${ }^{1}$ Also at: Department of Physics, Yunnan University, Kunming, P.R. China
} 
diation regions are thin in the longitudinal direction. Their double-peaked $\gamma$-ray structure was from two topologically disconnected outer gaps, each of which is associated with different magnetic poles. However, Romani and his co-workers (Chiang \& Romani, 1992,1994; Romani \& Yadigaroglu 1995; Romani 1996) have shown that only one outer gap with only outgoing current can already produce a double-peaked $\gamma$-ray light curve in a three-dimensional outer-magnetosphere. Using either a static dipolar field or a rotating dipolar field (results did not differ qualitatively), plus effects of time travel and aberration, they showed that an assumed outgoing current alone could produce a broad, irregularly-shaped emission beam which is particularly dense near the edge. Two $\gamma$-ray peaks would then be observed when the line of sight from the Earth crossed these enhanced $\gamma$-ray beam regions; the inner region of the beam provided a significant amount of emission between the peaks. EGRET has accumulated enough photons for Crab, Vela, PSR B1706-44, and Geminga to enable us to analyze the phase-resolved emission characteristics such as pulsed profiles and phaseresolved spectra (Fierro 1995; Thomson et al. 1996; Fierro et al. 1998). These data can give very strong constraint in theoretical models.

We reconsider the three-dimensional magnetosphere, following the important ground-breaking work of Romani and co-workers. But instead of assuming a single outer gap with only an outgoing current and no restriction on azimuthal directions, we use various physical processes (including pair production which depends sensitively on the local electric field and the local radius of curvature, surface field structure, and reflection of $e^{ \pm}$pairs because of mirroring and resonant scattering) to determine the three-dimensional geometry of the outer gap. In our model, two outer gaps and both outgoing and incoming currents are in principle allowed, but it turns out that outgoing currents dominate the emitted radiation intensities.

\section{Pair Production and the Structure of the Outer Gaps}

We propose a structure of outer gaps determined by pair production conditions. The potential drop of the gap is

$$
\Delta V \approx 6.6 \times 10^{12} f_{0}^{2} B_{12} P^{-2} \text { volts, }
$$

where $f_{0}=h(\langle r\rangle) R_{L}, h(\langle r\rangle)$ is the average vertical separation of the gap boundaries in the $(\boldsymbol{\Omega}, \mu)$ plane, $R_{L}=c / \Omega$ is the light cylinder radius, and $\langle r\rangle$ is the average distance to the gap-its value depends on the magnetic inclination angle $\chi\left(\langle r\rangle \sim R_{L} / 2\right)$. The particle current passing through the gap is

$$
\dot{N}_{g a p}=3 \times 10^{30} f_{0} \xi B_{12} P^{-2} \quad \mathrm{~s}^{-1},
$$

where $\xi=\Delta \Phi / 2 \pi ; \Delta \Phi$ is the transverse ( $\phi$-direction) extension of the gap. Each of the charged particles inside the gap will radiate high-energy curvature photons with a characteristic energy

$$
E_{\gamma}\left(f_{0}\right)=2 \times 10^{8} f_{0}^{3 / 2} B_{12}^{3 / 4} P^{-7 / 4} \mathrm{eV} .
$$

About half of $\dot{N}_{g a p}$ will move toward the star. Although they continue to radiate their energies on the way to the star, they still carry $10.5 P^{1 / 3}$ ergs of energy 
onto the stellar surface. The energy will be radiated back out in hard X-rays. However, resonant scattering with pairs near the star may reflect hard X-rays back to the stellar surface (Cheng, Gil, \& Zhang 1998; Wang et al. 1998), to be re-emitted as soft X-rays with a temperature

$$
T_{s} \approx 3.8 \times 10^{6} f_{0}^{1 / 4} \xi^{1 / 4} B_{12}^{1 / 2} P^{-5 / 12} \quad \mathrm{~K} .
$$

The X-ray photon density is very low, but each pair produced by X-ray curvature photon collisions in the outer gap will emit almost $10^{5}$ curvature $\gamma$ rays for further pair creation in that gap. Once the pair production threshold condition $k T_{s} E_{\gamma} \geq\left(m_{e} c^{2}\right)^{2}$ is satisfied, the gap is unlikely to grow much larger. This pair production condition gives

$$
f_{0}=5.5 P^{26 / 21} B_{12}^{-4 / 7} \xi^{1 / 7}
$$

Here, $\xi$ is still an unknown quantity. However, we see that $f_{0}$ is weakly dependent on $\xi$, which is likely of order of unity. In first approximation, we assume $f_{0}=$ $5.5 P^{26 / 21} B_{12}^{-4 / 7}$ (Zhang \& Cheng 1997). To determine $\xi$, we need to consider local pair production processes. The pair production per unit length inside the gap is a decreasing function of $r$. According to Cheng, Ho, \& Ruderman (CHR I; CHR II), $E_{\|} \propto r^{-1 / 2}$ for the thin outer gap (e.g., the Crab pulsar), which gives $E_{\gamma}(r) \propto r^{-1 / 8}$ after using the large $r$ limit $s(r)=\left(r R_{L}\right)^{1 / 2}$. Since $E_{\gamma}$ is only weakly dependent on $r$, we assume $\sigma_{\gamma \gamma} \approx$ constant.

The local pair production per unit length is

$$
N_{e^{ \pm}}(r)=\left(1-e^{-\tau_{\gamma \gamma}}\right) N_{\gamma}(r) \approx \tau_{\gamma \gamma} N_{\gamma}(r),
$$

where $\tau_{\gamma \gamma}=n_{X}(r) \sigma_{\gamma \gamma} l(r)$ is the local optical depth, $n_{X}=R^{2} T_{s}^{4} \sigma / r^{2} k T_{s} c$ is the X-ray number density at $r, l(r) \approx\left(2 s(r) f(r) R_{L}\right)^{1 / 2}$ is the local optical path, $f(r)=h(r) / R_{L}$ is the local vertical extension of the gap (since $B(r) h^{2}(r)$ is a constant, which gives $f(r) \propto r^{3 / 2}$ and $\left.f_{0} \sim f\left(R_{L} / 2\right)\right)$, and $N_{\gamma}=e E_{\|}(r) / E_{\gamma}(r)$ is the number of curvature photons emitted at $r$ per $e^{+} / e^{-}$per unit length. Then,

$$
N_{e^{ \pm}}(r) \propto r^{-11 / 8}
$$

We see that most pairs are produced near the null surface where $r=r_{i n}$. We estimate that the pair production will take place mainly in the range $r_{\text {in }} \leq r \leq$ $r_{\text {lim }}$, where $r_{l i m}$ is estimated as $r_{l i m} N_{e^{ \pm}}\left(r_{l i m}\right) / r_{i n} N_{e^{ \pm}}\left(r_{i n}\right) \sim\left(r_{l i m} / r_{i n}\right)^{-\overline{3} / 8} \sim$ $1 / 2$, which gives $r_{\text {lim }} \sim 6 r_{i n}$. This limits pair production both along the field lines and in transverse directions, and gives

$$
\Delta \Phi \sim 160^{\circ} .
$$

Within the pair production regions, outgoing and incoming directions for particle flows are allowed. For $r>r_{\text {lim }}$, only outgoing current is possible. Figure 1 shows our 3D outer-magnetic gap structure. Two pencil beams represent radio beams. The bulging spheroid is the surface of last-closed magnetic field lines. The two darker surfaces represent the upper boundaries of two outer gaps, so the structure of the outer gap starts from the null surface and ends at the light 


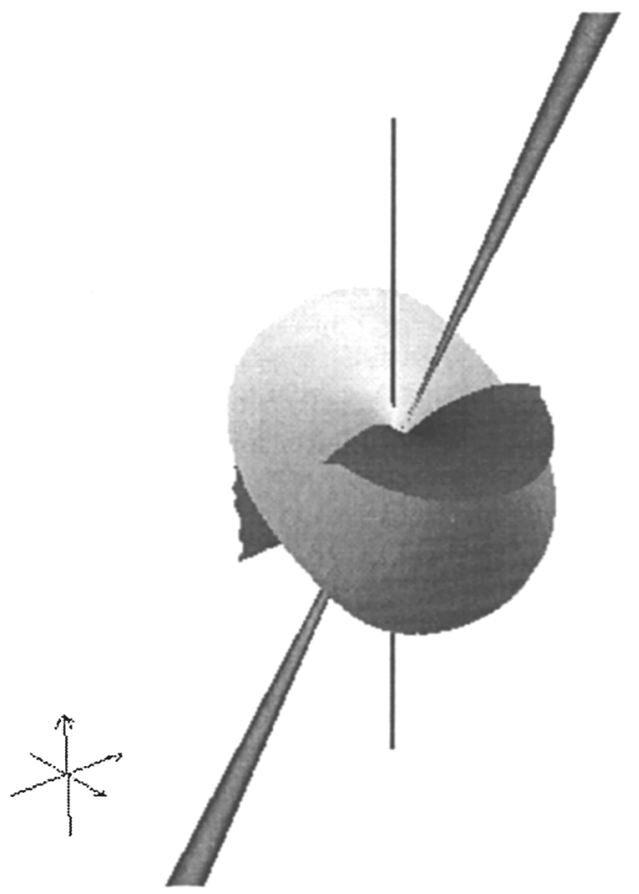

Figure 1. The three-dimensional outer-magnetic gap structure.

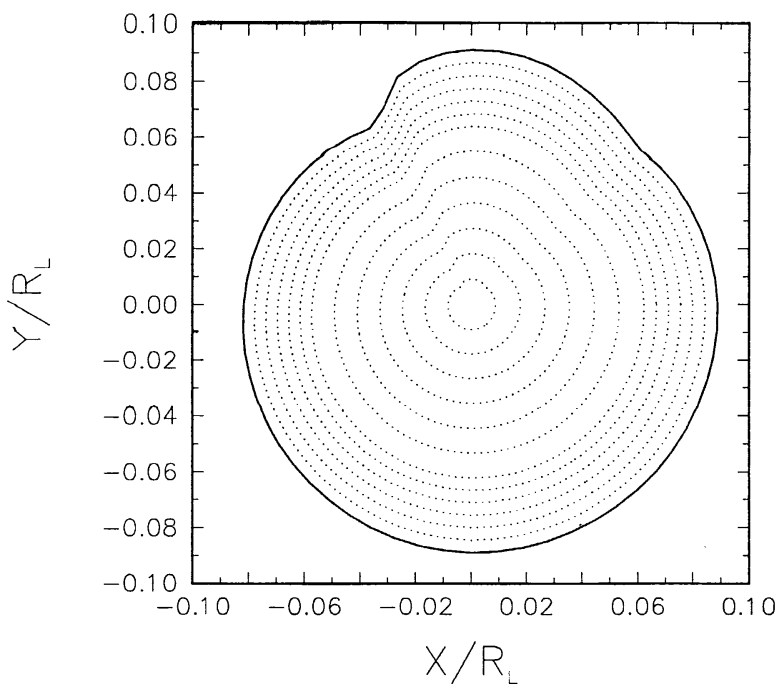

Figure 2. Example of a coordinate grid for the open volume. We define $a_{1}=1.0$ for the last closed line; $a_{1}$ changes by 0.05 from 1.0 to 0.7 , and by 0.1 from 0.7 to 0.1 . 
cylinder. The lower boundary is the last-closed field surface and the upper boundary is shown in Figure 1.

The polar cap shape defines the boundary of the open volume at the stellar surface. Because the outer gaps are within the open volume, we divide the open volume into many parts, in which the shape of each part at the stellar surface is the same as the polar cap shape but the size is smaller. First, we determine the coordinate values $\left(x_{0}, y_{0}, z_{0}\right)$ of the last-closed field lines at the stellar surface. Then, the coordinate values $\left(x_{0}^{\prime}, y_{0}^{\prime}, z_{0}^{\prime}\right)$ for different parts can be determined by using $x_{0}^{\prime}=a_{1} x_{0}, y_{0}^{\prime}=a_{1} y_{0}$, and $z_{0}^{\prime}=\left(1-\left(x_{0}^{\prime 2}+y_{0}^{\prime 2}\right)\right)^{1 / 2}$, and by changing $a_{1}$. In Figure 2, the open volume divided into these many parts is shown.

\section{Pair Production Beyond the Outer Gap Boundary}

Although pair production inside the gap is limited to a small region, pair production outside the gap is not restricted to a small region if synchrotron photons produced by secondary pairs exceed the thermal photons from the surface. The former photons do not get into the gap because of field line curvature (cf. CHR I), but they could convert most of the curvature photons from the gap into secondary pairs. We estimate the mean free path of curvature photons in the synchrotron $\mathrm{X}$-ray photon field as follows: the $\gamma$-ray mean free path $\lambda_{X \gamma} \sim\left(L_{X}\left(2 f_{0}\right) E_{\gamma} \sigma_{p} / l^{2} c(\mathrm{MeV})^{2}\right)^{-1} \sim 10^{7}\left(f_{0} / 0.1\right)^{-1} L_{36}^{-1} l_{8}^{2}\left(E_{\gamma} / 10 \mathrm{GeV}\right)^{-1} \mathrm{~cm}$, where $L_{36}$ is the X-ray luminosity in units of $10^{36} \mathrm{erg} \mathrm{s}^{-1}, l_{8}$ is the characteristic length to the outer gap in units of $10^{8} \mathrm{~cm}, E_{\gamma}$ is the typical energy of curvature radiation photons, and $\sigma_{p}$ is the pair production cross section. Here, we have considered that the threshold energy of pair production is $E_{X} E_{\gamma} \geq$ $(\mathrm{MeV})^{2} /\left(1-\cos \theta_{X \gamma}\right)$ and $\theta_{X \gamma} \sim\left(2 f_{0}\right)^{1 / 2}$. Since $l \ll R_{L}$, most curvature photons emitted from the accelerator will become secondary pairs outside the gap in some pulsars where the local magnetic field near the outer gap accelerator is sufficiently strong to give $\mathrm{KeV}$ synchrotron radiation.

\section{Photon Emission Morphologies}

As pointed out above, in this model, two outer gaps can exist in the open field line volume of the pulsar magnetosphere (cf. Figure 1). Due to pair production, outer gaps are limited along both the radial and azimuthal directions. Therefore, the model allows both outward and inward emission, but inward emission is only from the region $\left(r_{\text {lim }}-r_{i n}\right)$ of the outer gaps. A large fraction of high-energy photons emitted inwards cannot pass freely through the inner magnetosphere within a certain distance of the star where these photons are converted into secondary $e^{ \pm}$pairs by the intense magnetic field. These secondary pairs will emit lower-energy photons through their synchrotron radiation. Some of those synchrotron photons will be further converted into pairs. Moreover, about half of the charged particles accelerated in the outer gap will move inwards along magnetic field lines. These charged particles will produce high-energy curvature radiation photons. These curvature photons can be converted into pairs near the star. A cascade will develop there until the Lorentz factors of the pairs approach unity. Nonthermal X-rays can be produced in this cascade, and thermal X-rays 


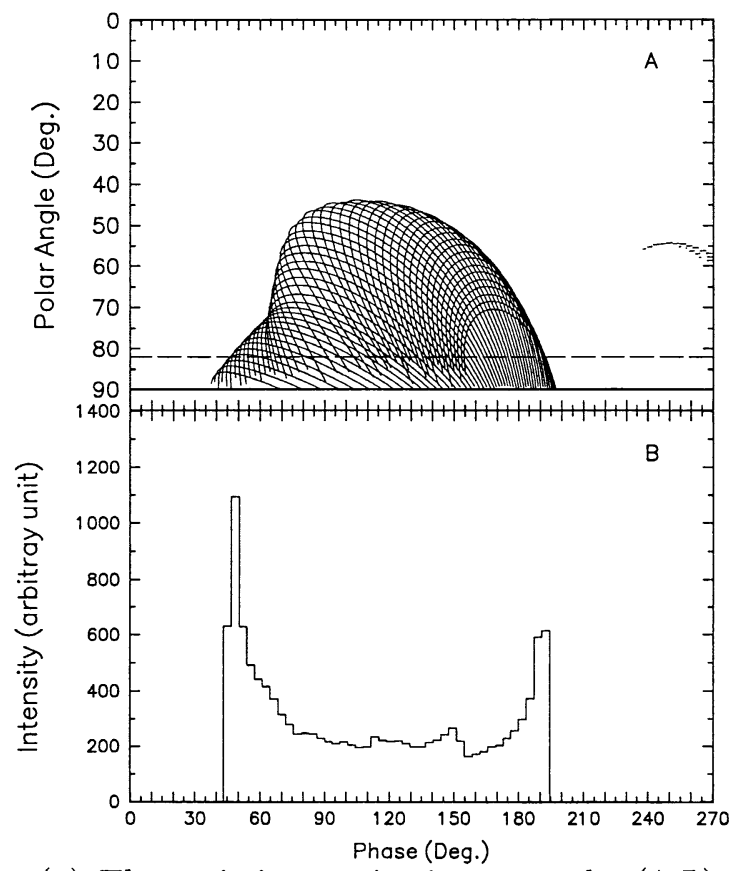

Figure 3. (a) The emission projection onto the $(\zeta, \Phi)$ plane $(a 1=$ $0.9)$, and (b) the corresponding pulse profile $(\Delta a 1=0.03)$. The emission consists of the emission outwards from both outer gaps and inwards only from the region $\left(r_{l i m}-r_{i n}\right)$ of both outer gaps. The outer gaps are limited along the azimuthal direction by pair production. The Crab parameters, $\alpha=65^{\circ}, \zeta=82^{\circ}$, and $\delta \phi=160^{\circ}$, are used.

can be radiated from the stellar surface (Halpern \& Ruderman 1993; Zhang \& Cheng 1997; Cheng et al. 1998; Wang et al. 1998; Cheng \& Zhang 1999). In panel A of Figure 3, the photon emission from a single outer gap is shown in the $(\zeta, \Phi)$ plane, where $\zeta$ is the polar angle from the rotation axis and $\Phi$ is the phase of rotation of the star. Part of the inward emission which can pass freely through the inner magnetosphere is also shown (dashed curves). The pulse profile corresponding to the emission pattern of panel A of Figure 3 is shown in panel B of Figure 3.

\section{Phase-resolved Spectra of the Crab Pulsar}

From the emission morphologies, we see that photons emitted into a given phase come from different positions of the outer gap. Figure 4 shows the trajectory of the emission region for various pulsar phases for a given viewing angle. Since curvature radiation, synchrotron radiation, and inverse Compton scattering depend on local quantities, e.g., curvature, $E_{\|}, B, n_{p h}$, etc., it is likely that the radiation spectrum varies with phase. Figure 5 shows observed, phase-resolved data of the Crab pulsar, which are clearly phase-dependent. First, the primary 


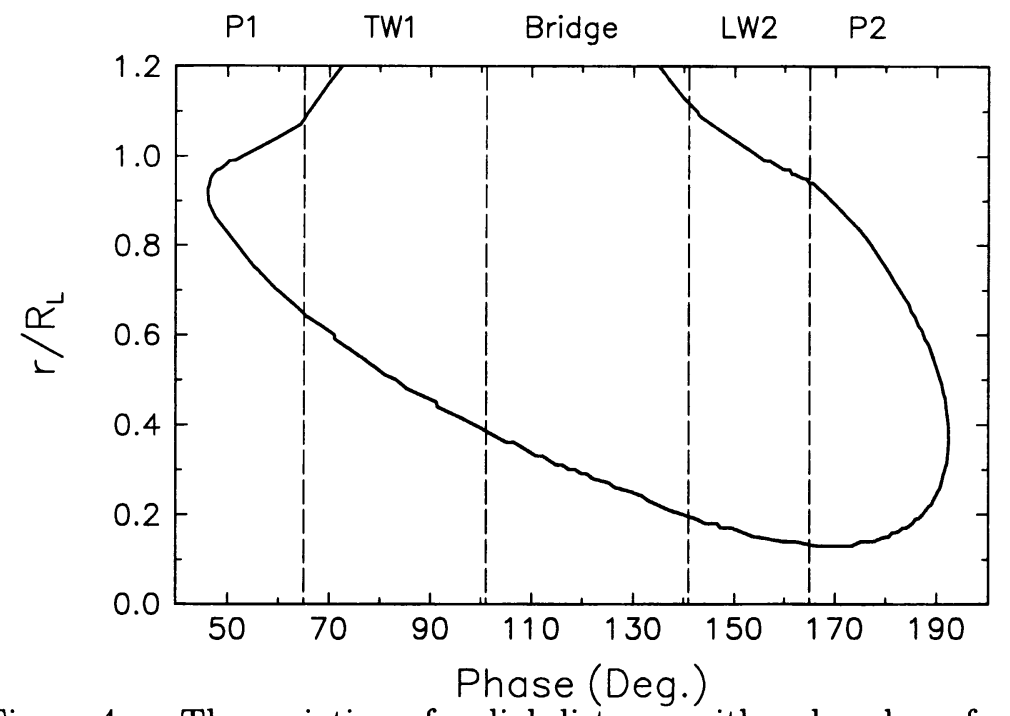

Figure 4. The variation of radial distance with pulse phase for the Crab. The inclination angle is $65^{\circ}$, and $a_{1}=0.9$. Five regions for different pulse phases are indicated.

charged particles lose their energy via curvature radiation with a spectrum

$$
\frac{d^{2} \dot{N}_{\gamma}(r)}{d V d E_{\gamma}}=\frac{l_{c u r}(r) n_{G J}(r)}{E_{c u r}(r)} \frac{1}{E_{\gamma}} \quad \text { for } \quad E_{\gamma} \leq E_{c u r},
$$

where $l_{c u r}(r)=e E_{\|}(r) c$ is the local power in curvature radiation produced by a single $e^{+} / e^{-}, n_{G J}$ is the local Goldreich-Julian number density, and $E_{c u r}(r)$ is the local curvature radiation energy. In Section 4, we pointed out that, in many $\gamma$-ray pulsars, most curvature photons will be converted into pairs by the synchrotron photons of the secondary pairs. The secondary pairs should then have the distribution

$$
\frac{d N_{e^{ \pm}}(r)}{d E_{e}} \approx \frac{1}{\dot{E}_{e}} \frac{l_{c u r}(r) n_{G J}(r)}{E_{c u r}(r)} \ln \left(\frac{E_{c u r}}{E_{e}}\right) \Delta V(r),
$$

where $\dot{E}_{e}$ is the rate of energy loss of the secondary pairs and $\Delta V(r)$ is the involved volume at position $r$. The radiation mechanisms of the secondary pairs include synchrotron radiation and inverse Compton scattering. Figures 5 are the comparisons between model results and the phase-resolved spectra of the Crab pulsar. In obtaining our model results, we have used one single surface in the calculation despite the fact that the emission region of secondary pairs has finite thickness. The local pitch angle of the secondary pairs is approximated by $\sin \beta(r) \approx l(r) / s(r)$, where $l(r)$ and $s(r)$ are the local mean free path and the local radius of curvature, respectively. The calculated, phase-averaged spectrum of the Crab pulsar and the comparison with the observed data is shown in Figure 6 . 

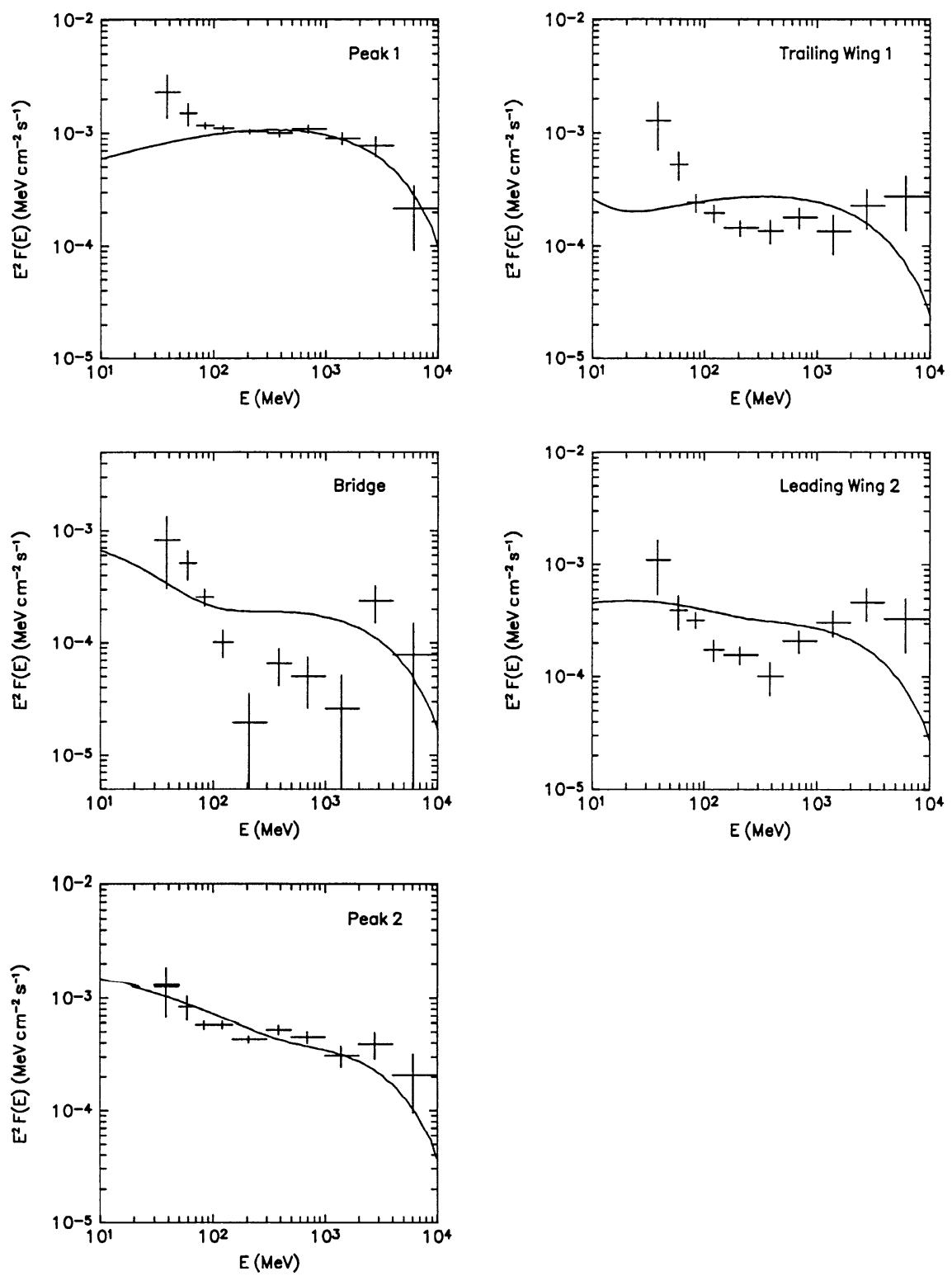

Figure 5. Phase-resolved $\gamma$-ray spectra for different phases (peak 1, trailing wing 1 , bridge, leading wing 2 , and peak 2) of the Crab pulsar. Observed data are taken from Fierro et al. (1998). 


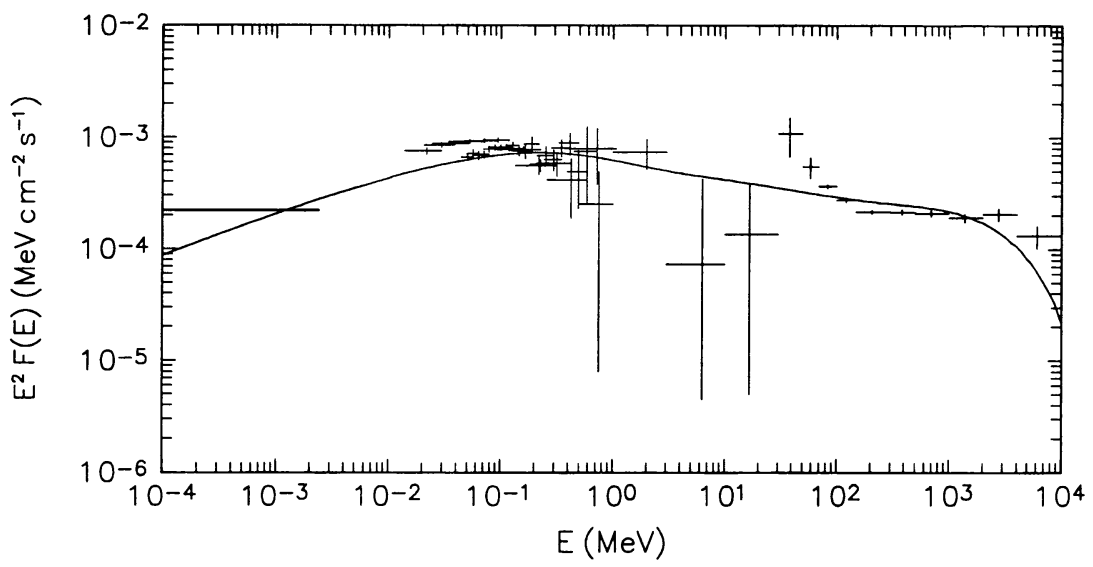

Figure 6. Phase-averaged spectrum for the Crab. The observed ROSAT data is from Becker \& Trümper (1997). The observed OSSE, BATSE, and COMPTEL data are taken from Ulmer et al. (1995). The EGRET data is from Fierro et al. (1998).

\section{Summary and Discussion}

We use a 3D model magnetosphere to give the observed light curve and the phase-resolved spectra of the $\gamma$-ray pulsars. In our model, the local photonphoton pair production in the outer gaps limits the extension of the outer gaps along the azimuthal direction. We find that the two topologically disconnected outer gaps, with some extension along the azimuthal $(\phi)$ direction, exist in the pulsar magnetosphere. Double-peaked pulse profiles with varying phase separation, depending on viewing angle, and strong bridge emission occur naturally, as in the single pole outer gap model. Although pair production inside the outer gap is only limited to a small region, the intense $\mathrm{X}$-rays produced by secondary pairs in the outer-magnetosphere of the Crab pulsar can convert most curvature photons into pairs outside the gap. The observed Crab pulsar spectrum of the Crab pulsar results from a synchrotron/self-Compton mechanism. We have applied our model to explain the Crab pulsar's phase-resolved spectra, and the results are consistent with observation. In low-energy parts of P1, TW1, Bridge, and LW2 of the phase-resolved spectra of EGRET, the observed data are higher than those of the model calculation, which implies that the synchrotron component is stronger than the model calculation. In the high-energy parts of the phase-resolved spectra, the data are also higher than the model results. We speculate that some of the primary curvature photons escape conversion by the secondary X-rays. Therefore, a curvature component with a typical energy $\sim 10 \mathrm{GeV}$ shows up in the high-energy tail of the observed phase-resolved spectra. There are some improvements which can be made in our model. In our calculations, only one surface layer of the magnetic field is used, and photons are assumed to be emitted tangent to the local field lines. Therefore, the emission 
light curves (cf. Figure 3 ) have sharp edges in both ends. Some of the secondary pairs have quite large pitch angles, and their emission will not be tangent to the local field lines. In future calculations, these should be taken into account. Furthermore, the relative phases between X-rays and $\gamma$-rays can be calculated as well. Finally, we point out that our calculation is based on a steady-state accelerator. The realistic outer-magnetospheric gap may be a dynamical or flickering one.

Acknowledgments. We thank R.W. Romani for useful discussion and suggestions, P.L. Nolan for providing us EGRET data, and M.P. Ulmer for OSSE, BASTE, and COMPTEL data of the Crab pulsar. This work is partially supported by an RGC grant of the Hong Kong Government.

\section{References}

Cheng, K. S., Gil, J., \& Zhang, L. 1998, ApJ, 493, L35

Cheng, K. S., Ho, C., \& Ruderman, M.A. 1986a, ApJ, 300, 500 (CHR I)

Cheng, K. S., Ho, C., \& Ruderman, M.A. 1986b, ApJ, 300, 522 (CHR II)

Cheng, A. F., Ruderman, M. A., \& Sutherland, P.G. 1976, ApJ, 203, 209

Cheng, K. S., \& Zhang, L. 1999, ApJ, 515, 337

Chiang, J., \& Romani, R.W. 1992, ApJ, 400, 724

Chiang, J., \& Romani, R.W. 1994, ApJ, 436, 754

Fierro, J. M. 1995, Ph.D. Thesis, Stanford University

Fierro, J. M., Michelson, M., Nolan, P. L., \& Thomson, D. J. 1998, ApJ, 494, 734

Halpern, J. P., \& Ruderman, M. 1993, ApJ, 415, 286

Holloway, N. J. 1973, Nature Physical Science, 246, 6

Romani, R. W. 1996, ApJ, 470, 469

Romani, R. W., \& Yadigaroglu, I.-A. 1995, ApJ, 438, 314

Thompson, D. J., et al. 1996, ApJ, 465, 385

Ulmer, M. P., et al. 1995, ApJ, 448, 356

Wang, F. Y.-H., Ruderman, M., Halpern, J. P., \& Zhu, T. 1998, ApJ, 498, 373

Zhang, L., \& Cheng, K. S. 1997, ApJ, 487, 370

Zhu, T., \& Ruderman, M. 1997, ApJ, 478, 701 